\title{
Phase three studies of biologics for severe asthma: could do better?
}

\author{
Douglas S. Robinson ${ }^{1}$, Harsha H. Kariyawasam ${ }^{1,2}$ and Liam G. Heaney ${ }^{3}$ \\ Affiliations: 'Severe Asthma Service, Respiratory Medicine, UCLH NHS Foundation Trust, London, UK. \\ ${ }^{2}$ Specialist Adult Allergy and Clinical Immunology Dept, Rhinology Section, Royal National Throat Nose Ear \\ Hospital, London, UK. ${ }^{3}$ Centre for Experimental Medicine, School of Medicine, Dentistry and Biomedical \\ Sciences, Queen's University Belfast, Belfast, UK.
}

Correspondence: Douglas S. Robinson, Severe Asthma Service, Respiratory Medicine, 4th Floor East, 250 Euston Road, London NW1 2PG, UK.E-mail: douglas.robinsonduclh.nhs.uk

@ERSpublications

We need to reconsider how we assess biologics for severe asthma http://ow.ly/j7Mw30efCHb

Cite this article as: Robinson DS, Kariyawasam HH, Heaney LG. Phase three studies of biologics for severe asthma: could do better? Eur Respir J 2017; 50: 1701108 [https://doi.org/10.1183/13993003.01108-2017].

It is an exciting time for those of us treating patients with severe asthma. We now have more effective ways of assessing patients, monitoring adherence, and defining airway inflammation [1-5]. Current trials should inform on the role of biomarkers in determining treatment response [6], as we move away from a one-size-fits-all approach with oral prednisolone as the best alternative available. We can now select patients for intervention with biologics.

As we have previously stressed, protocolised assessment including objective measures of adherence are essential before categorising someone as having severe asthma [7, 8]. In much of Europe and the UK, care is delivered through standardised assessment protocols at defined specialist services linked to research units $[1,2]$. We now have licensed biologics targeting key inflammatory pathways: IgE with omalizumab and eosinophils with mepolizumab, with over 10 years clinical experience with the former, and extensive trial data with the latter [9-11]. A surprising feature of omalizumab in clinical practice has been that, unlike for most drugs, outcomes in clinics [12] have often been better than those seen in clinical trials [9]. In the context of severe asthma, selection of patients for treatment with biologics may be more rigorous than for trials, since in many settings payers have set the bar high and require full protocolised assessment, and frequent oral prednisolone courses, in addition to treatment of comorbidities and confirmation of treatment adherence. Future trials should only select protocol-assessed patients who remain symptomatic despite optimisation of management, or who require long term oral prednisolone.

One can learn the importance of treating the right patient with the right drug from the mepolizumab studies. It took many years to define the subgroup of patients with severe asthma who respond to anti-IL-5 treatment: in retrospect it is not a great surprise that those with residual eosinophilia (detectable in blood or sputum) respond, while those who are not eosinophilic do not $[10,11,13,14]$. Defining the relevant clinical outcome, specifically eosinophilic exacerbations, was also a critical part of defining the benefit of this class of therapy in severe disease. However, this concept of subtypes of asthma is new and clinically still evolving. Much has been made of endotypes, with many different pathways described as potentially contributing to the asthma phenotype [15]. There has been much debate about type 2 (IgE and eosinophilia driven by IL-4, IL-5 and IL-13) and non-type 2 asthma [16, 17]. Whether real non-type 2 asthma exists in the severe asthma population is still an open question: some patients have symptoms despite high dose inhaled steroids without residual type 2 inflammation, but the precise mechanism of

Received: June 012017 | Accepted: June 222017

Conflict of interest: Disclosures can be found alongside this article at erj.ersjournals.com

Copyright @ERS 2017 
persistent symptoms in this group is unclear. The current RASP-UK study adjusting therapy according to type 2 biomarkers, exhaled nitric oxide, blood eosinophils and serum periostin (an IL-13 induced epithelial protein) should help define which patients have controlled type 2 disease, and which (if any) have a non-type 2 mechanism driving their symptoms [6].

Recent publications have shown benefit in large clinical trials of anti-IL-5 approaches with mepolizumab $[10,11,14]$, reslizumab [18] and benralizumab [19, 20] (all target interleukin-5), and dupilumab (anti-IL4/IL-13) [21]. However, in all studies with exacerbation rates and/or oral steroid reduction as outcomes there were very large placebo effects. Large phase III studies of lebrikizumab (anti-IL-13) did not show significant clinical benefit compared to placebo [22]. Of note, the lebrikizumab phase 3 studies did not enrich for exacerbations by requiring a pre-specified exacerbation history in the previous year [22].

We would argue there are important lessons for future development of "add-on" therapies to standard optimised care for severe asthma.

Firstly, with the availability of licenced anti-IgE and anti-IL-5 therapy, it is becoming difficult to argue ethically that in studies of these "add-on" therapies for patients who remain uncontrolled despite optimised standard care with high dose inhaled steroids with or without additional controllers, one could treat one arm with placebo. Companies, regulators, clinicians and patients need to consider whether comparator studies should be performed with the currently best available treatment including biologic therapies (as is the case for new cancer treatments or rheumatogical diseases).

Secondly, it is notable that the current large phase III studies tend to recruit from many sites, which may not all be specialist severe asthma centres, and it is unclear whether all patients have had detailed assessment, management of co-morbidities and objective measures of adherence. There have been a number of studies which have highlighted the comorbidity and complexity of a difficult-to-manage asthma population [1-5]. Most published studies have made little or no mention of other organ involvement or effects on common comorbidities, despite the systemic effect of treatment. In particular, co-existent nasal symptoms from rhinitis, rhinosinusitis or nasal polyps have seldom been assessed, despite separate trials suggesting potential benefit $[23,24]$. This may reflect regulatory rules, but that is not an excuse of not considering all aspects of our patients' illness. Importantly, we should also be aware that current dosing for these products is extrapolated from the clinical trials and there may be room for more personalised treatment regimens depending on defined clinical or inflammatory features of disease and individual patient responses.

Thirdly, many phase III trials in severe asthma have been designed to show a reduction in exacerbations in a type-2 biomarker high population and consistently these studies enrich for both relevant (or what is predicted as relevant) biomarker profile and a history of prior exacerbations. Adherence studies in severe asthma have consistently shown a higher exacerbation rate in poorly adherent patients [25] and our suspicion is that less than ideally optimised asthma management and medication adherence in large phase three studies accounts at least in part for the high placebo response.

Finally, when a drug is eventually licenced, payers impose additional criteria on access to restrict the population on the basis of questionable economic models. For example in the UK for both omalizumab and mepolizumab, a protocolised assessment at a severe asthma centre multidisciplinary team is required and only patients with four or more courses of oral prednisolone or long term oral prednisolone are eligible for reimbursement under criteria produced by the National Institute for Health and Care Excellence [26, 27]. All of these issues mean that the patient population for whom the drug is eventually made available may be very different from the population recruited to the pivotal clinical trials.

Unless there is a re-evaluation of how we perform phase III "add-on" clinical trials in asthma, we anticipate that it may become difficult to recruit to large trials, and the problems discussed above will increase. It is perhaps time to move towards detailed systematic clinical assessment and objective assessment of adherence before recruitment to clinical trials that will allow comparator studies with currently available and effective treatments in optimised patients. This would ideally be done during phase 2 development, using monitored adherence to inhaled treatment so that the true effect size of the "add-on" treatment can be estimated in patients with severe disease optimised on best standard of care therapies. This may also lead to a better estimate of the anticipated "placebo effect" in larger studies and consequently lead to smaller and more cost-effective studies in phase 3 by implementing the systematic clinical assessment and objective measurements of adherence developed in the phase 2 programme.

The emergence of biologics has led to optimism and excitement in this new era of severe asthma medicine: at last new treatments are available with the potential to transform the quality of life of our patients. We owe it to them to assess these novel agents effectively to optimise the benefits, avoiding over-optimistic expectations but also making sure we do not throw the baby out with the bath water! 


\section{References}

1 Robinson DS, Campbell DA, Durham SR, et al. Systematic assessment of difficult-to-treat asthma. Eur Respir J 2003; 22: 478-483

2 van der Meer AN, Pasma H, Kempenaar-Okkema W, et al. A 1-day visit in a severe asthma centre: effect on asthma control, quality of life and healthcare use. Eur Respir J 2016; 48: 726-733.

3 McNicholl DM, Stevenson M, McGarvey LP, et al. The utility of fractional exhaled nitric oxide suppression in the identification of nonadherence in difficult asthma. Am J Respir Crit Care Med 2012; 186: 1102-1108.

4 Jia G, Erickson RW, Choy DF, et al. Bronchoscopic Exploratory Research Study of Biomarkers in Corticosteroid-refractory Asthma (BOBCAT) Study Group. J Allergy Clin Immunol 2012; 130: 647-654.

5 Heaney LG, Conway E, Kelly C, et al. Predictors of therapy resistant asthma: outcome of a systematic evaluation protocol. Thorax 2003; 58: 561-566.

6 Heaney LG, Djukanovic R, Woodcock A, et al. Research in progress: Medical Research Council United Kingdom Refractory Asthma Stratification Programme (RASP-UK). Thorax 2016; 71: 187-189.

$7 \quad$ Heaney LG, Robinson DS. Severe asthma treatment: need for characterising patients. Lancet 2005; 365: 974-976. Robinson DS. Assessing severe asthma. Eur Respir J 2016; 48: 611-613.

9 Humbert M, Beasley R, Ayres J, et al. Benefits of omalizumab as add-on therapy in patients with severe persistent asthma who are inadequately controlled despite best available therapy (GINA 2002 step 4 treatment): INNOVATE. Allergy 2005; 60: 309-316.

10 Ortega HG, Liu MC, Pavord ID, et al. Mepolizumab treatment in patients with severe eosinophilic asthma. $N$ Engl J Med 2014; 371: 1198-1207.

11 Bel EH, Wenzel SE, Thompson PJ, et al. Oral glucocorticoid-sparing effect of mepolizumab in eosinophilic asthma. N Engl J Med 2014; 371: 1189-1197.

12 Niven RM, Saralaya D, Chaudhuri R, et al. Impact of omalizumab on treatment of severe allergic asthma in UK clinical practice: a UK multicentre observational study (the APEX II study). BMJ Open 2016; 6: e011857.

13 Flood-Page P, Swenson C, Faiferman I, et al. A study to evaluate safety and efficacy of mepolizumab in patients with moderate persistent asthma. Am J Respir Crit Care Med 2007; 176: 1062-1071.

14 Pavord ID, Korn S, Howarth P, et al. Mepolizumab for severe eosinophilic asthma (DREAM): a multicentre, double-blind, placebo-controlled trial. Lancet 2012; 380: 651-659.

15 Wenzel SE. Asthma phenotypes: the evolution from clinical to molecular approaches. Nat Med 2012; 18: 716-725.

16 Woodruff PG, Modrek B, Choy DF, et al. T-helper type 2-driven inflammation defines major subphenotypes of asthma. Am J Respir Crit Care Med 2009; 180: 388-395.

17 Robinson D, Humbert M, Buhl R, et al. Revisiting Type 2-high and Type 2-low airway inflammation in asthma: current knowledge and therapeutic implications. Clin Exp Allergy 2017; 47: 161-175.

18 Castro M, Zangrilli J, Wechsler ME, et al. Reslizumab for inadequately controlled asthma with elevated blood eosinophil counts: results from two multicentre, parallel, double-blind, randomised, placebo-controlled, phase 3 trials. Lancet Respir Med 2015; 5: 355-366.

19 FitzGerald JM, Bleecker ER, Nair P, et al. Benralizumab, an anti-interleukin-5 receptor $\alpha$ monoclonal antibody, as add-on treatment for patients with severe, uncontrolled, eosinophilic asthma (CALIMA): a randomised, double-blind, placebo-controlled phase 3 trial. Lancet 2016; 388: 2128-2141.

20 Bleecker ER, FitzGerald JM, Chanez P, et al. Efficacy and safety of benralizumab for patients with severe asthma uncontrolled with high-dosage inhaled corticosteroids and long-acting $\beta 2$-agonists (SIROCCO): a randomised, multicentre, placebo-controlled phase 3 trial. Lancet 2016; 388: 2115-2127.

21 Wenzel S, Castro M, Corren J, et al. Dupilumab efficacy and safety in adults with uncontrolled persistent asthma despite use of medium-to-high-dose inhaled corticosteroids plus a long-acting $\beta 2$ agonist: a randomised double-blind placebo-controlled pivotal phase 2 b dose-ranging trial. Lancet 2016; 388: 31-44.

22 Hanania NA, Korenblat P, Chapman KR, et al. Efficacy and safety of lebrikizumab in patients with uncontrolled asthma (LAVOLTA I and LAVOLTA II): replicate, phase 3, randomised, double-blind, placebo-controlled trials. Lancet Respir Med 2016; 4: 781-796.

23 Gevaert P, Calus L, Van Zele T, et al. Omalizumab is effective in allergic and nonallergic patients with nasal polyps and asthma. J Allergy Clin Immunol 2013; 131: 110-116.

24 Gevaert P, Van Bruaene N, Cattaert T, et al. Mepolizumab, a humanized anti-IL-5 mAb, as a treatment option for severe nasal polyposis. J Allergy Clin Immunol 2011; 128: 989-995.

25 Heaney LG, Horne R. Non-adherence in difficult asthma: time to take it seriously. Thorax 2012; 67: 268-270.

26 National Institute for Health and Care Excellence. Omalizumab for treating severe persistent allergic asthma. Technology appraisal guidance [TA278]. Date last updated: April 24, 2013.

27 National Institute for Health and Care Excellence. Mepolizumab for treating severe refractory eosinophilic asthma. Technology appraisal guidance [TA431]. Date last updated: January 25, 2017. 